\title{
Circadian Clues to Sleep Onset Mechanisms
}

\author{
Kurt Kräuchi, Dipl.Chem.HTL, and Anna Wirz-Justice, Ph.D.
}

Thermoregulatory processes have long been implicated in initiation of human sleep. A meta-analysis of studies carried out under the controlled conditions of a constant routine protocol followed by nocturnal sleep revealed that heat loss, indirectly measured by the distal-proximal skin temperature gradient, was the best predictor variable for sleep onset latency (compared with core body temperature or its rate of change, heart rate, melatonin onset, and subjective sleepiness ratings). The cognitive signal of "lights out" induced relaxation, with a consequent shift in heat redistribution from the core to the periphery (as measured by an abrupt increase in skin temperatures and a rapid fall in heart rate). These thermoregulatory changes took place before sleep onset: sleep itself had minor further effects. Thus, when the confounding, long-lasting masking effects of lying down are controlled for, circadian thermoregulation initiates sleep, but does not appear to play a major role in its maintenance.

[Neuropsychopharmacology 25:S92-S96, 2001]

(c) 2001 American College of Neuropsychopharmacology.

Published by Elsevier Science Inc.
KEY WORDS: Heat loss; Core body and skin temperatures; Melatonin; Heart rate; Sleepiness; Sleep onset latency

Human sleep research is (mostly) carried out during the night. Yet sleep is embedded in the 24-hour day, and its timing, duration, and internal structure is strongly determined by the circadian pacemaker. Thus, in order to investigate mechanisms initiating sleepiness and sleep propensity, measurement needs to begin long before the sun sets. Our chronobiological approach to the physiology underlying sleep onset focuses on the role of melatonin and thermoregulation.

In both night-active and diurnal species, the circadian peak of melatonin secretion occurs during the night (for review see: Arendt 1995). In contrast, all species, independent of temporal niche, sleep during the circadian trough of the core body temperature (CBT)

From the Centre for Chronobiology, Psychiatric University Clinic, Wilhelm Klein Strasse 27, CH-4025 Basel, Switzerland

Address correspondence to: Anna Wirz-Justice, Ph.D., Centre for Chronobiology, Psychiatric University Clinic, Wilhelm Klein Strasse 27, CH-4025 Basel, Switzerland, Tel.: 004161325 5473, Fax: 004161 325 5577, E-mail: anna.wirz-justice@unibas.ch rhythm. In rats, melatonin administration enhances norepinephrine-induced vasoconstriction of the tail (Viswanathan et al. 1990); in humans, melatonin induces vasodilation in fingers and toes (Kräuchi et al. 1998), suggesting that transduction of the nocturnal melatonin signal is linked to opposite physiological sequelae appropriate to behavioral niche. To further characterize the functional relationships preceding sleep onset, we have analyzed data from a series of experiments designed to phase-shift the circadian system with different putative zeitgebers, using identical methodology of a constant routine (CR) protocol to permit pooling of data.

\section{"CORE" AND "SHELL" THERMOREGULATION}

Nearly fifty years ago, Aschoff formulated in detail the two-component thermoregulatory concept of "core" and "shell" (Aschoff 1956). The core is homeostatically regulated around $37^{\circ} \mathrm{C}$. However, the shell is not; it depends largely on the environmental temperature, and can be considered poikilothermic, like a lizard. In a hot environment the shell is small; in a cold environment it 
is large, and thus acts as a buffer to protect the core from dangerous cooling. Normally, heat loss is regulated by the cardiovascular system: cardiac output, and whether blood vessels are constricted or dilated. The two components of the shell are the distal skin regions, e.g. hands and feet, and proximal skin regions, e.g. the trunk. Body heat is transported from the core to the skin of the shell mainly by convective blood flow. A specialized thermoregulatory system is anatomically localized only in distal skin regions: the arteriovenous anastomoses (AVAs). They act as a shunt for rapid blood flow from the arterioles directly to the dermal venous plexus enabling rapid heat exchange: when AVAs are open, this is about 10,000 times faster than capillary blood flow (Hales 1985). Heat loss occurs mainly through the skin by this mechanism under normal conditions (enhanced under more extreme conditions such as sweating).

It is well known that the setpoint of the thermoregulatory system is not constant throughout the day; it undergoes a characteristic circadian modulation driven by the circadian pacemaker in the suprachiasmatic nuclei (Kittrell 1991). Given this temporal program, how is this translated into a certain temperature at a certain time of day? CBT results from the balance between heat production and heat loss. Aschoff could show that CBT declines when heat loss exceeds heat production in the evening (at the beginning of the sleep period), and vice versa in the morning (Aschoff and Heise 1972). We could confirm this finding under the reduced masking conditions of a CR protocol (continuous wakefulness for 34 h; Kräuchi et al. 1998). CBT showed the well known endogenous circadian rhythm, rising to a peak in the late afternoon with a trough during the second half of the night. Proximal skin regions with only slow capillary blood flow followed the CBT rhythm. Distal skin temperature showed an inverse time course, presumably regulated by opening the AVAs to initiate heat loss during the night. There was an additional small secondary increase in the afternoon. A similar pattern was found for the distal-proximal skin gradient (DPG). The DPG provides a rather selective measure for thermoregulatory skin blood flow through AVAs (and consequent heat loss), while adjusting for (i.e. subtracting) changes in capillary blood flow, represented by skin temperature changes at proximal regions (Rubinstein and Sessler 1990). Heat production (indirect calorimetry) was low during the night, and rose very swiftly in the morning to a maximum (Kräuchi et al. 1998). It appeared to have a bimodal rhythm, with a second, lesser trough in the afternoon. Melatonin followed the inverse time course of the CBT rhythm. Thus, the endogenous circadian rhythm of CBT results from a balance between heat production and heat loss. The next question is which of these thermoregulatory processes is related to increased sleepiness and sleep propensity.

\section{THERMOREGULATION AND SLEEPINESS}

Sleep is usually initiated on the declining portion of the CBT curve in the evening (Czeisler et al. 1980; Zulley et al. 1981). It is most likely to occur when CBT is declining at its maximum rate (Campbell and Broughton 1994). Hypothermia induced by administration of melatonin or benzodiazepines is associated with increased subjective sleepiness and reduced sleep onset latency (Gilbert et al. 1999; Kräuchi et al. 1998). However, a simple comparison of three small daytime CR studies could link sleepiness to vasodilation of distal skin regions rather than the decline of CBT per se. After postural change from an upright to a supine position, CBT declined and foot skin temperature rose together with sleepiness (Kräuchi et al. 1998). The opposite occurred during an orthostatic challenge (Kräuchi et al. 1998). In contrast, when ice was eaten (Figure 1), although CBT also declined abruptly, this did not induce sleepiness, rather, alertness was augmented, together with a reduced distal skin temperature as well as DPG, i.e. vasoconstriction (Kräuchi et al. 1999b). A decline in CBT is therefore a consequence of heat loss, not a primary cause of sleepiness induction.

In order to reveal the best predictor for sleep onset latency (SOL) among diverse variables (skin tempera-

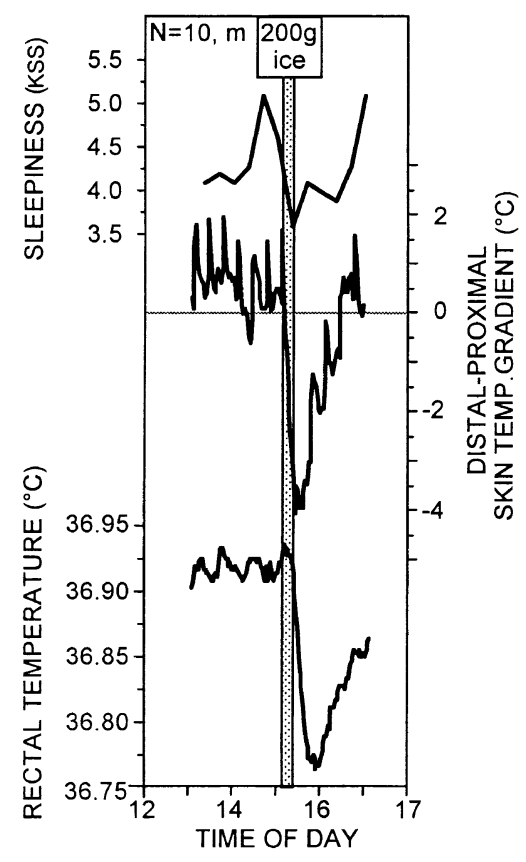

Figure 1. Mean time course of subjective sleepiness (Karolinska Sleepiness Scale), distal (thumb)-proximal (forearm) skin temperature gradient and core (rectal) body temperature before and after ingestion of $200 \mathrm{~g}$ crushed ice. Note: distal vasoconstriction occurred immediately on ice ingestion, whereas the decrease in core body temperature followed with a time lag. In contrast, subjective sleepiness did not increase (Kräuchi et al. 1999b). 
tures, CBT or its rate of change, heart rate, melatonin onset, and subjective sleepiness ratings) a backward stepwise regression analysis revealed the highest correlations of SOL with DPG in the 1.5-h episode before lights out; all the other potential predictor variables were not included in the regression model (Kräuchi et al. 1999a, 2000). Therefore, under our controlled experimental conditions it is an increase of distal vasodilation, and hence heat loss, which goes hand in hand with induction of sleepiness, and in turn with reduced sleep onset latency.

\section{DOES SLEEP REDUCE TEMPERATURE?}

Information about the more subtle effect of "lights out" - a cognitive signal to relax and fall asleep - also emerged from this CR protocol. The large dataset and the controlled CR conditions long before sleep onset without any postural changes permitted detailed analysis of whether any of the above thermoregulatory variables changed predictably before or after sleep onset. Even though the time course of skin temperature in proximal and distal regions is opposite during wakefulness, and remains so throughout the $34 \mathrm{~h}$ of a sleep deprivation (Kräuchi et al. 1998), it can follow similar patterns when both capillaries and AVAs are similarily constricted or dilated (Kräuchi et al. 2000). Such a pattern is initiated already at lights out, even before sleep onset. What happens to these thermoregulatory functions in the evening and night is shown in Figure 2.

At lights out and hence, implicit "permission" to sleep, both proximal and distal skin temperatures immediately increase, heart rate decreases abruptly, and CBT declines slowly. These phenomena reflect the shift in heat redistribution from core to periphery when totally relaxed. However, when awake in a $C R$, even though supine, a slight vasoconstriction remains. These differences may explain why CBT at night reaches a lower minimum during sleep than the endogenous circadian rhythm when awake.

It has been recognized for many years that relaxation leads to a significant decrease in cutaneous sympathetic nerve activity and, in turn, to enhanced peripheral blood flow (Baker et al. 1976; Kleitman 1987; Noll et al. 1994; Velluti 1997). When the data in the left panel are aligned to sleep onset instead of lights out, it is clear that the thermoregulatory changes have already taken place (Kräuchi et al. 2001). It appears that when the confounding long-lasting effects of lying down are controlled for, only minor changes in thermoregulation occur after sleep onset: all the functionally important mechanisms have already been set in motion to initiate, but not necessarily to maintain sleep.
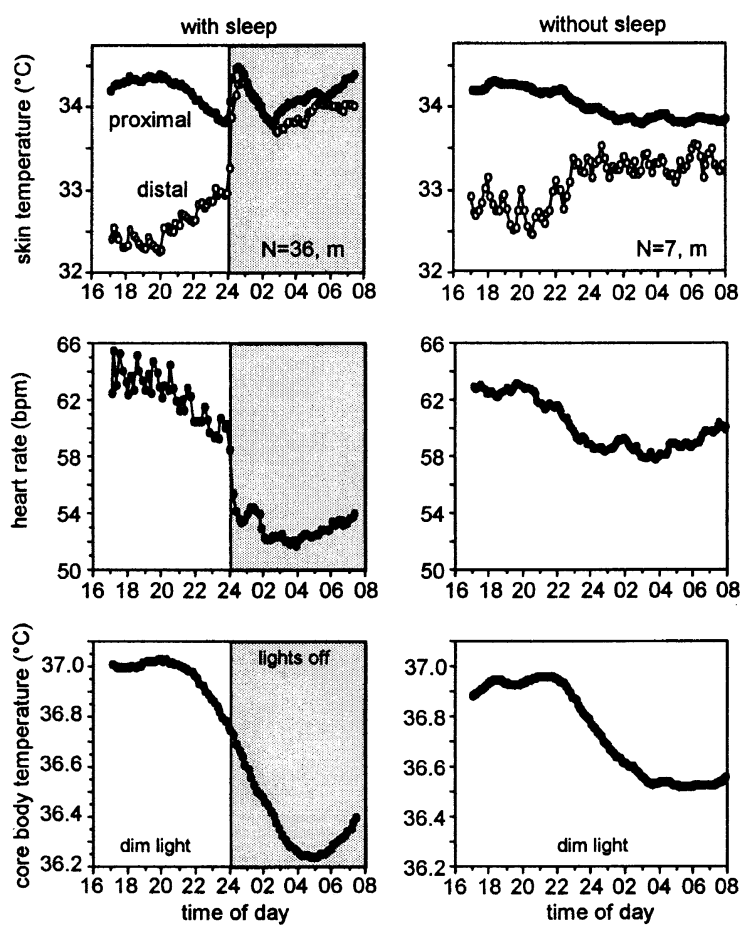

Figure 2. Comparison of the time course of distal and proximal skin temperatures, heart rate, and core (rectal) body temperature (CBT) in two protocols where sleep was permitted (left panels) or not (right panels). Note: distal and proximal skin temperature increased in parallel after lights out. However, without sleep, proximal skin temperature followed the CBT rhythm. Heart rate and CBT dropped to a lower value during sleep (Kräuchi et al. 1998, 2000).

\section{THERMOREGULATION AND SLEEP: A CIRCADIAN MECHANISM}

Sleep has been claimed to cause changes in thermoregulation: our analyses suggest that these assumptions may need to be reversed. It required the stringent conditions of a constant routine protocol many hours before lights out to dissect out the time course of thermoregulatory events leading to sleep onset. Although CBT globally correlates with sleep onset latency, it is not the key factor. The best predictor for a short sleep onset latency is vasodilated distal skin regions (Kräuchi et al. 1999a; Kräuchi et al. 2000). Heat loss initiation via the onset of nocturnal melatonin secretion may be the mechanism underlying the circadian regulation of sleep propensity ("opening the sleep gate", Tzischinsky and Lavie 1994). Sleep follows, but does not cause thermoregulatory changes. Slow wave sleep or slow wave activity has minor, if any, thermoregulatory function (Kräuchi et al. 2001).

Under usual conditions, diverse behaviors (warm bath, hot drink, lying down, lights out, relaxation, sex) act as "positive masking" to prepare the body for sleep: all induce vasodilation. Their timing is usually coinci- 
dent with the circadian decline in CBT and melatonin onset, which represent the output of the circadian pacemaker (process " $\mathrm{C}$ " in the 2-process model of sleep regulation (Borbély 1982)). Therefore, both these "helpful masking effects" and the circadian regulation of core body temperature and melatonin induce similar thermoregulatory changes, i.e. heat loss, which may represent the physiological correlate for the natural increase in sleepiness at night. Since a total sleep deprivation during a constant routine protocol also increases sleepiness, but without any significant thermoregulatory changes (Kräuchi et al. 1998), this suggests that homeostatically regulated sleepiness and sleep propensity (process "S" in the 2-process model of sleep regulation (Borbély 1982)) is different from the above thermoregulatory-related sleepiness.

These findings have important clinical implications. As recognized by everyone who has camped outdoors, it is difficult to fall asleep with cold feet. Indeed, cold feet and the inability to vasodilate may be a physiological cause of some sleep disturbances, particularly in the elderly (Ancoli-Israel et al. 1986; Pache et al. 2001, Van Someren 2000). In a single case study, both problems were treated with biofeedback techniques (AncoliIsrael et al. 1986). If distal vasodilation indeed represents a physiological "final common pathway", this mechanism may underlie the sleep-inducing effects of the hot bath and the hot toddy, phytotherapies, benzodiazepines, and other somnifers. The direct effects of such sleeping aids could also thereby possibly override a malfunctioning or maltimed biological clock.

\section{ACKNOWLEDGMENTS}

This paper is dedicated to Dr. J. Christian Gillin, colleague and long-time friend. AWJ was the Harold L. Dorris Neurological Research Center Visiting Professor at The Scripps Research Institute during the preparation of this manuscript. The Basel studies were supported by the Swiss National Science Foundation and carried out in collaboration with Drs. Christian Cajochen, Esther Werth, and Konstantin Danilenko.

\section{REFERENCES}

Ancoli-Israel S, Selfert AR, Lemon M (1986): Thermal biofeedback and periodic movements in sleep: patients' subjective reports and a case study. Biofeedback SelfRegul 11:177-188

Arendt J (1995): Melatonin and the mammalian pineal gland. London, Chapman \& Hall

Aschoff J (1956): Wechselwirkungen zwischen Kern und Schale im Wärmehaushalt. Arch Physikalische Therapie 3:113-133

Aschoff J, Heise A (1972): Thermal conductance in man: its dependence on time of day and of ambient tempera- ture. In Itoh S, Ogata K, Yoshimura H (eds), Advances in Climatic Physiology. Tokyo, Igako Shoin, pp 334-348

Baker MA, Cronin MJ, Mountjoy DG (1976): Variability of skin temperature in the waking monkey. Am J Physiol 230:449-455

Borbély AA (1982): A two-process model of sleep regulation. Hum Neurobiol 1:195-204

Campbell SS, Broughton RJ (1994): Rapid decline in body temperature before sleep: fluffing the physiological pillow? Chronobiol Int 11:126-131

Czeisler CA, Weitzman ED, Moore-Ede MC, Zimmerman JC, Knauer RS (1980): Human sleep: its duration and organization depend on its circadian phase. Science 210:1264-1267

Gilbert SS, van den Heuvel CJ, Dawson D (1999): Daytime melatonin and temazepam in young adult humans: equivalent effects on sleep latency and body temperatures. J Physiol 514:904-914

Hales JRS (1985): Skin arteriovenous anastomoses, their control and role in thermoregulation. In Johansen K, Burggren WW (eds), Cardiovascular Shunts. Alfred Benzon Symposium 21. Copenhagen, Munksgaard, pp 433-451

Kittrell EMW (1991): The suprachiasmatic nucleus and temperature rhythms. In Klein DC, Moore RY (eds), Suprachiasmatic Nucleus: The Mind's Clock. New York, Oxford University Press, pp 233-245

Kleitman N (1987): Sleep and Wakefulness. Chicago, The University of Chicago Press

Kräuchi K, Cajochen C, Werth E, Renz C, von Arb M, WirzJustice A (2001): Thermoregulatory changes occur after lights off not after onset of sleep stage 2. Sleep 24 (Suppl):A165-A166

Kräuchi K, Cajochen C, Werth E, Wirz-Justice A (1999a): Warm feet promote the rapid onset of sleep. Nature 401:36-37

Kräuchi K, Cajochen C, Werth E, Wirz-Justice A (2000): Functional link between distal vasodilation and sleeponset latency? Am J Physiol 278:R741-R748

Kräuchi K, Cajochen C, Wirz-Justice A (1998): Circadian and homeostatic regulation of core body temperature and alertness in humans: what is the role of melatonin? In Honma KI, Honma S (eds), Circadian Clocks and Entrainment, Vol 7. Sapporo, Hokkaido University Press, pp 131-146

Kräuchi K, Werth E, Wüest D, Renz C, Wirz-Justice A (1999b): Interaction of melatonin with core body cooling: sleepiness is primarily associated with heat loss and not with a decrease in core body temperature. Sleep 22 (Suppl 1):S285-S286

Noll G, Elam M, Kunimoto M, Karlsson T, Wallin BG (1994): Skin sympathetic nerve activity and effector function during sleep in humans. Acta Physiol Scand 151:319-329

Pache M, Kräuchi K, Cajochen C, Wirz-Justice A, Dubler B, Flammer J, Kaiser HJ (2001): Cold feet and prolonged sleep-onset latency in vasospastic syndrome. The Lancet 358:125-126

Rubinstein EH, Sessler DI (1990): Skin-temperature gradients correlate with fingertip blood flow in humans. Anesthesiology 73:541-545

Tzischinsky O, Lavie P (1994): Melatonin possesses timedependent hypnotic effect. Sleep 17:638-645 
Van Someren EJ (2000): More than a marker: interaction between the circadian regulation of temperature and sleep, age-related changes, and treatment possibilities. Chronobiol Int 17:313-354

Velluti RA (1997): Interactions between sleep and sensory physiology. J Sleep Res 6:61-77
Viswanathan M, Laitinen JT, Saavedra JM (1990): Expression of melatonin receptors in arteries involved in thermoregulation. Proc Natl Acad Sci USA 87:6200-6203

Zulley J, Wever R, Aschoff J (1981): The dependence of onset and duration of sleep on the circadian rhythm of rectal temperature. Pflügers Arch 391:314-318 